\title{
Pulse Mapping for Impulse Radio Ultra Wideband Communications
}

\author{
Zhan Yu $^{1} \quad$ Ping Luo $^{1} \quad$ Suguru Fujita ${ }^{2}$ \\ 1 Panasonic Singapore Laboratories Pte. Ltd. \\ Blk 1022 Tai Seng Ave \#06-3530, Singapore $534415 \quad$ Matsushita Electronics Industrial Co. Ltd, Tokyo, Japan \\ Email: Raymond.Yuz@sg.panasonic.com.sg
}

\begin{abstract}
Recently, Ultra Wideband (UWB) has become attractive as an ultra high-speed transmission technique in WPAN. Impulse Radio (IR) UWB promises to bring a low power consumption and low cost. Effective mapping methods for a high-order IR modulation have been not considered yet, although a high-order IR modulation should be employed to a ultra high-speed wireless link above 1 Giga-bit per second (Gbps). In this paper, a novel Hamming distance enlarging pulse mapping method is proposed to IR UWB systems. It is presented by using an exemplary modulation scheme and its mapping table that the proposed mapping method can achieve a maximum Hamming distance between all neighboring pulses. A larger Hamming distance can increase the correction ability of forward error codes and improve the system BER performance. The effectiveness of proposed pulse mapping method to the conventional Gray mapping method was also demonstrated in a computer simulation.
\end{abstract}

\section{Introduction}

Impulse Radio (IR) is a new transmission technology for wireless personal area networks (WPAN) by using ultra short carrierless pulses, where the pulse width is typically in the order of nanosecond. The pulses are transmitted at low power but with a broad bandwidth more than several GHz [1][2]. This technology has many advantages which stem from its broad bandwidth nature.

In US, a several GHz of bandwidth (microwave: 3.1$10.6 \mathrm{GHz}$ ) has been authorized by FCC for license-free Ultra-WideBand (UWB) WPAN at a low radiated power [3]. This is much larger than the bandwidths of other contemporary wireless technologies and allows for high data rate wireless communications. Hence, UWB technology currently can provide wireless connectivity among personal devices in the universal serial bus (USB) interface which supports 480 Mbps.

Furthermore, millimeter-wave (mmW) band is expected to realize an ultra high speed link above Giga bit per second (Gbps). In US, a license-free ISM band (57-64GHz) is allowed to adopt for short range communication systems defined by FCC 47 CFR 15.255
[4]. In Japan, an unlicensed band is also allocated from 59 to $66 \mathrm{GHz}$ as well as in US. The mmW systems can realize long range transmission and multipath robustness, because a small size directional antenna can be easily employed in the $\mathrm{mmW}$ transceiver. IR-mmW has potential to provide ultra high speed communications among audio visual consumer products like uncompressed High Definition video streaming.

IR system can commonly employ pulse-polarity modulation, pulse-amplitude modulation (PAM), phase modulation, pulse-position modulation (PPM) or $M$-ary versions of these. In IR systems, a high-order modulation is desired in order to achieve a high data-rate transmission, such as 1Gbps. For a high-order modulation, a pulse mapping method becomes essential and has a significant effect on the overall system performance. The conventional Gray mapping method [5][6] is designed to have only one digital bit change between a desired pulse and its neighboring pulses. The neighboring pulse is defined here as the pulse which has a smallest difference in amplitude, pulse position or phase from the desired pulse.

Due to channel noises and multipath fading, the detection errors occur at the receiver mostly by detecting neighboring pulses instead of the desired pulse. In general, forward error correction (FEC) codes at channel encoder are used to correct such detection errors.

The Gray mapping has a nice performance when there is no FEC code involved. However, when FEC code is used, the Gray mapping cannot achieve the optimum performance. This is because the Gray mapping doesn't explore the error correction ability of FEC codes and the Hamming distance between neighboring pulses is only one. Hamming distance is defined for the number of binary bit difference. A larger Hamming distance is required in FEC codes for correcting more detection errors. Therefore, there exists a need to have a better pulse mapping method to maximize the Hamming distance between all neighboring pulses in IR systems with channel coding.

In this paper, a novel pulse mapping method for highorder modulation IR systems is proposed. Applying this method, one can maximize the Hamming distance between the desired pulse and all neighboring pulses, furthermore the proposed method is easy to realize and 
can be applied into any IR modulation technique.

The remainder of the paper is organized as follows. Section 2 introduces the signal model. The proposed pulse mapping method is presented in Section 3. The simulation results are presented in Section 4. Conclusions are drawn in Section 5.

\section{System Model}

\subsection{IR Transmitter}

Figure 1 shows a typical block diagram of an IR UWB transmitter. The IR transmitter comprises a channel encoder, an interleaver, an IR modulator with a pulse mapping table, a pulse generator, a code generator and a transmitting antenna.

The information bits represent input messages, which could be any one or combination of text, video, image, audio, etc. All kinds of FEC codes can be adopted in the channel encoder. Convolutional coding and block coding are the two major forms of channel coding. Convolutional codes operate on serial data, one or few bits at a time. Block codes operate on relatively large (typically up to a couple of hundred bytes) message blocks. Thus, block codes always have a longer waiting time than convolutional codes.

The code generator is employed at the transmitter to randomize the pulse position or pulse polarity of the modulated signal for multiple access applications. For example, a time-hopping (TH) pseudo noise (PN) code is used in [2] to randomize the pulse position of PPM signal at each pulse repetition frame (PRF).

The pulse generator can produce the IR signal at particular pulse position or with particular pulse polarity based on the PN code. The pulse waveform is also another important parameter which should follow the power spectrum density (PSD) requirement of the transmitted signal, such as monocycle pulse waveform in [2]. In UWB, the FCC sets the PSD mask with a maximum value $-41.3 \mathrm{dBm} / \mathrm{MHz}$ over the frequency band from $3.1 \mathrm{GHz}$ to $10.6 \mathrm{GHz}$. Finally, the IR signal can be sent by the transmitting antenna.

\subsection{IR Receiver}

Figure 2 shows a typical block diagram of an IR UWB receiver. The receiver comprises a receiving antenna, a low noise amplifier, a pulse generator, a code generator, an IR demodulator with a pulse mapping table, a deinterleaver and a channel decoder.

At the receiver, the IR demodulator is generally a correlator by multiplying the received signal with a local template signal from a pulse generator and integrating the signal after multiplication. The integration result can be used for demodulation as in [7]. A RAKE receiver also can be adopted in IR systems to achieve the optimum detection over multipath channels.

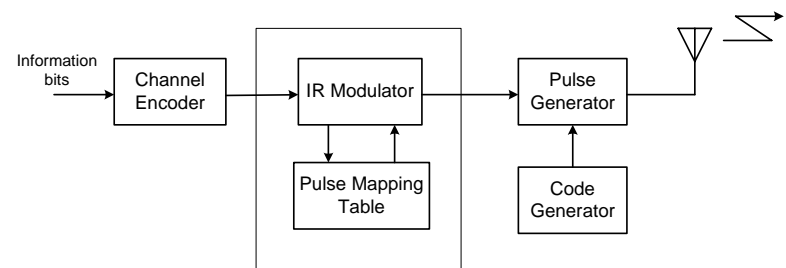

Figure 1 A typical Impulse Radio UWB transmitter

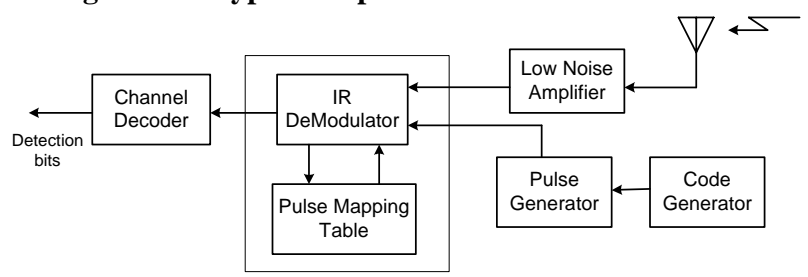

Figure 2 A typical Impulse Radio UWB receiver

\subsection{IR Modulation}

The PPM and PAM are two common modulation schemes for IR systems. If the PPM modulation is employed in IR systems, the transmitted IR signal can be shown as follows [2][7],

$$
S_{P P M}(t)=\sum_{j=-\infty}^{+\infty} p\left(t-j T_{f}-c_{j} T_{c}-d_{j} \varepsilon\right)
$$

where $p(\cdot)$ represents the transmitted pulse waveform. $T_{f}$ is the frame duration or pulse repetition time, where the bit duration $T_{B}=N_{f} T_{f}$ and $N_{f}$ is the number of frames for one bit. $T_{c}$ is the chip duration. The $\mathrm{TH}$ sequence $\left\{c_{j}\right\}$ are all integer elements in the range of $0 \leq c_{j}<N$. It is assumed that $N T_{c}+d_{j} \varepsilon<T_{f} . \varepsilon$ is the time shift between different pulse positions in PPM and $d_{j}$ is the position level in PPM.

If the PAM modulation is employed in IR systems, the transmitted IR signal can be shown as follows [7],

$$
S_{\text {PAM }}(t)=\sum_{j=-\infty}^{+\infty} a_{j} p\left(t-j T_{f}\right)
$$

where $a_{j}$ is the amplitude level in PAM and all $a_{j}$ 's are selected from a group of predetermined amplitude levels. If the group has only two amplitude levels which having the same absolute value but different polarities, such binary-PAM is also called "pulse polarity modulation".

The PPM and PAM also can be combined in IR modulation. Then the transmitted IR signal can be shown as,

$$
S_{P P M \& P A M}(t)=\sum_{j=-\infty}^{+\infty} a_{j} p\left(t-j T_{f}-c_{j} T_{c}-d_{j} \varepsilon\right)
$$

Another implementation to IR modulation is known as transmitted-reference (TR) scheme [8][9]. In the TR scheme, there is a reference pulse transmitted before each data pulse. The reference pulse has a fixed amplitude 
level or time position. Then the modulation can be carried out on the polarity, amplitude or time position of data pulse relative to the reference pulse. For example, a TR pulse interval amplitude modulation (PIAM) was proposed in [10] to modulate both polarity and time position of data pulse relative to the reference pulse.

\subsection{Pulse Mapping}

For a high-order modulation with more than one bit per symbol, the mapping table becomes important to determine time position, amplitude or phase of pulses in the IR modulation. The conventional Gray mapping method in [5][6] is designed to have only one digital bit change between a desired pulse and its neighboring pulses. The neighboring pulse is defined here as the pulse which has a smallest difference in amplitude, pulse position or phase from the desired pulse. It is equivalent to the neighboring symbols with smallest Euclidean distance in conventional carrier-based systems.

In Figure 3, a combination of 4-PPM and 2-PAM is adopted as an example to show the conventional Gray mapping method. It is assumed in (3) that $a_{j}$ can have two different amplitude levels $\{A,-A\}$ and $d_{j}$ can have four position levels $\{1,2,3,4\}$. Thus totally there are eight different modulated pulses, A, B, ..., H in Figure 3 and they can represent 3 incoming binary bits.

As shown in Figure 3, some pulses, such as A, B, G and $\mathrm{H}$, have two neighboring pulses. Other pulses, such as $\mathrm{C}, \mathrm{D}, \mathrm{E}$ and $\mathrm{F}$, have three neighboring pulses. For example, the pulse $\mathrm{A}$ has two neighboring pulses $\mathrm{B}$ and $\mathrm{C}$, where pulse $\mathrm{B}$ has the same pulse position as the pulse $\mathrm{A}$ but a minimum difference in pulse amplitude and pulse $\mathrm{C}$ has the same pulse amplitude as the pulse $\mathrm{A}$ but a minimum difference in pulse position. The pulse $\mathrm{E}$ has three neighboring pulses $\mathrm{C}, \mathrm{G}$ and $\mathrm{F}$, where both pulses $\mathrm{C}$ and $\mathrm{G}$ has the same pulse amplitude as the pulse $\mathrm{E}$ but a minimum difference in pulse position and pulse $\mathrm{F}$ has the same pulse position as the pulse $\mathrm{E}$ but a minimum difference in pulse amplitude.

One possible Gray mapping table is presented in Figure 3. Based on the Gray mapping table, all the neighboring pulses in Figure 3 have only one binary bit difference between each other. Therefore, the Hamming distance between neighboring pulses is equal to 1 .

\section{Hamming distance enlarging pulse mapping method}

The conventional Gray mapping method doesn't explore the error correction ability of FEC codes, since the Hamming distance between all neighboring pulses is only one. A larger Hamming distance can support FEC codes to correct more detection errors at the receiver. In the TR-PIAM [10], although the Hamming distance between

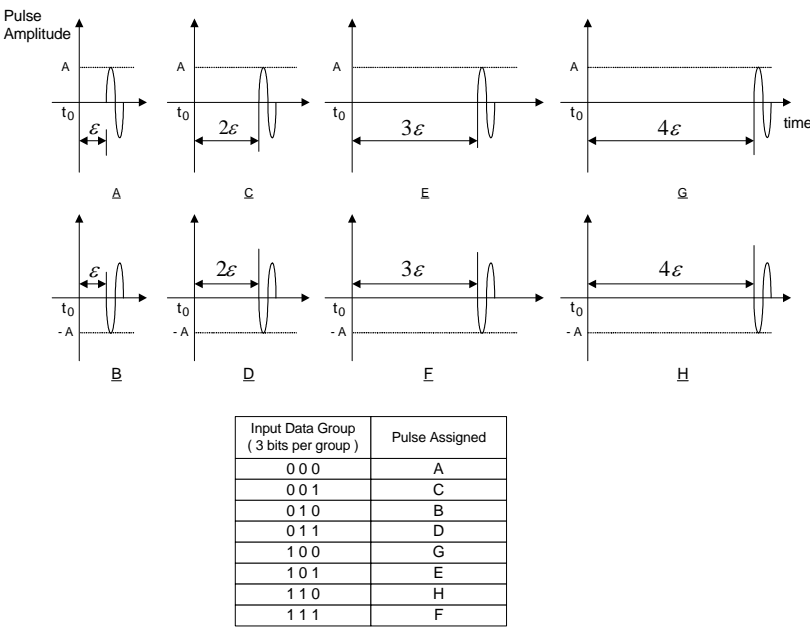

Figure 3 Gray mapping method for a combination of 4PPM and 2-PAM

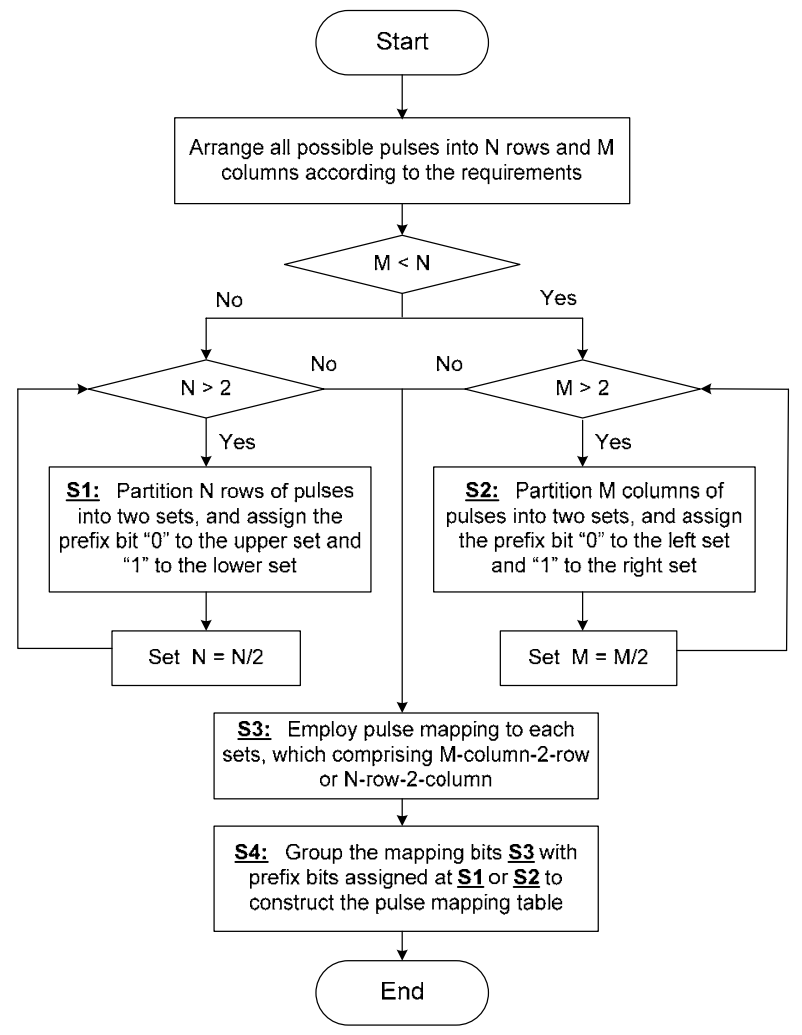

Figure 4 Flow chart of Hamming distance enlarging pulse mapping for a combination of $M$-PPM and $N$-PAM

the neighboring pulses with opposite polarity is maximized, the Gray mapping is still used between the neighboring pulses with different time positions. Hence, the TR-PIAM cannot provide large Hamming distance when the detection errors occurred by time position distortion, which commonly happen in dense multipath or interference environments.

In [6], Trellis-Coded Modulation (TCM) can be 
found to maximize the Hamming distance and Euclidean distance between neighboring symbols. But both modulations are designed for carrier-based bandwidthconstrained communication systems, which are quite different from IR systems.

In this chapter, a Hamming distance enlarging pulse mapping method is presented for a combination of $M$-ary PPM and $N$-ary PAM. $M$ and $N$ are the modulation levels for PPM and PAM, respective. In this paper, it is assumed that $M \geq 2, N \geq 2$ and both $\mathrm{M}$ and $\mathrm{N}$ are the integer powers of 2 .

In Figure 4, a flow chart of the proposed Hamming distance enlarging pulse mapping method is present for $M$-ary PPM and $N$-ary PAM. At the flow chart, the first step is to arrange all possible pulses into a specific matrix structure: all the pulses with same amplitude are grouped at the same row with a decreasing order of amplitude from top to bottom in; all the pulses with same time position are grouped at the same column with an ascent order of time delay from left to right.

Then a comparison between $M$ and $N$ is made. If $M \geq N$, the partition on $N$ rows is carried out; however if $M<N$, the partition on $M$ columns is carried out. The purpose of this comparison is to minimize the operational complexity. Since the partition stops when either $N$ or $M$ reaches 2, the number of partitions can be reduced by carrying out the partition on the smaller one of $M$ and $N$.

If $M \geq N$ and $N>2, N$ rows are partitioned equally into two halfs or sets at $\underline{\mathrm{S} 1}$. A first prefix bit "0" is assigned to the upper set and " 1 " is assigned to the lower set. Then the value $N$ is replaced by $N / 2$, and the partition keeps running until $N=2$. If $N>M$ and $M>2, M$ columns are partitioned equally into two halfs or sets at $\mathbf{S} 2$. The similar prefix bit assignment is carried out on $M$ columns, and the only difference is $M$ columns are divided into left and right halfs or sets. After the matrix partition stops, all the remaining sets are either $M$ column-2-row or $\mathrm{N}$-row-2-column. For both cases, the same partition method is carried out to each set at $\underline{\mathrm{S}}$.

In order to have a clear presentation for steps $\underline{\mathrm{S} 1}, \underline{\mathrm{S} 2}$ and $\underline{S} 3$ of the flow chart, a combination of 8-PPM and 4PAM is adopted as an example for illustration in Figure 5. Firstly, a matrix of pulses with 8 columns and 4 rows can be constructed in (a) by arranging amplitudes $A_{1}>A_{2}>A_{3}>A_{4}$ and positions $t_{1}<t_{2}<\cdots<t_{8}$. The pulse amplitudes at the same row are identical, and the pulse positions at the same column are identical. In the matrix, each pulse has either 3 or 4 neighboring pulses based on its location.

Since $M \geq N$ and $N>2$, the matrix is partitioned

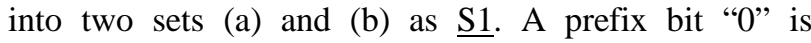
assigned to upper set (a) and " 1 " is assigned to lower set (b). The number of rows in each set is equal to 2 , so the partitioning $\underline{\mathrm{S} 1}$ stops. Both sets are the same and the pulse mapping $\underline{\mathrm{S} 3}$ for each set is identical, so only the pulse mapping for set (b) is presented in Figure 5. As shown in Figure 5, the set (b) is partitioned into two groups by separating odd and even columns. After that, two groups are further divided into subgroups by a cross manner. Then at $\underline{\mathrm{S} 3}$, all 16 pulses are ranged at one row, and $\log _{2}(M N / 2)=4$ bits are assigned to each pulse from left to right in an ascending order, which is " 0000 ", "0001", ..., and " 1111 " as (c). Finally at $\underline{\mathrm{S} 4}$, all the mapping bits at $\underline{\mathrm{S} 3}$ are grouped with prefix bits assigned

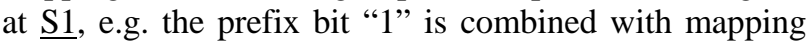
bits (c) to create (d) for set (b) in Figure 5. The proposed pulse mapping table for the combination of 8-PPM and 4PAM can be completely constructed as in Table 1 .

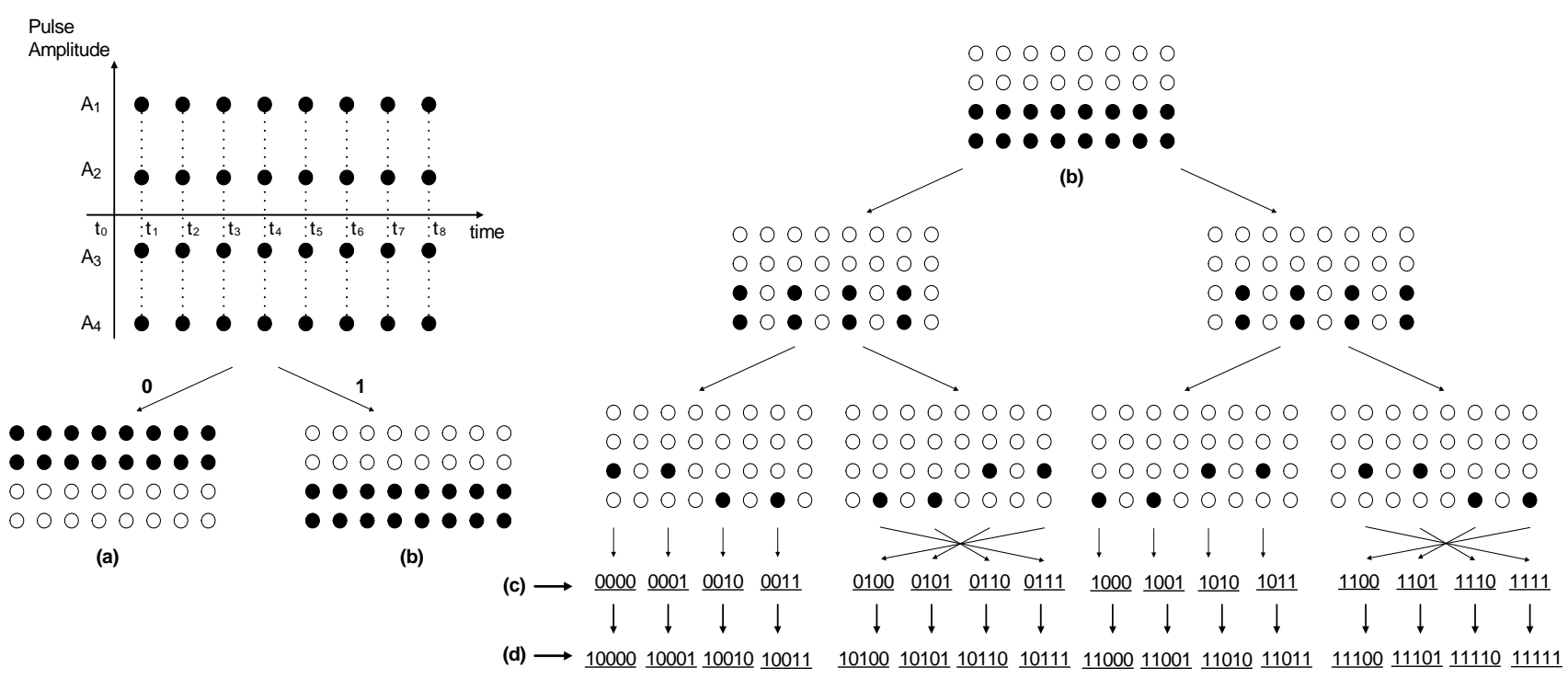

Figure 5 Proposed Hamming distance enlarging pulse mapping method for a combination of 8-PPM and 4-PAM 
Table 1 Proposed and Gray pulse mapping tables

\begin{tabular}{|c|c|c|c|c|c|c|c|}
\hline \begin{tabular}{|l} 
Input Data Bits \\
( 5 bits per \\
symbol $)$
\end{tabular} & \begin{tabular}{|c|} 
Assigned \\
Amplitude \& \\
Time position
\end{tabular} & \begin{tabular}{|l} 
Input Data Bits \\
( 5 bits per \\
symbol $)$
\end{tabular} & \begin{tabular}{|c|} 
Assigned \\
Amplitude \& \\
Time position
\end{tabular} & \begin{tabular}{|l} 
Input Data Bits \\
( 5 bits per \\
symbol $)$
\end{tabular} & \begin{tabular}{|c|} 
Assigned \\
Amplitude \& \\
Time position
\end{tabular} & \begin{tabular}{|l} 
Input Data Bits \\
( 5 bits per \\
symbol $)$
\end{tabular} & \begin{tabular}{|c|c|} 
Assigned \\
Amplitude \& \\
Time position
\end{tabular} \\
\hline 00000 & A1 \& $\mathrm{t} 1$ & 01000 & A2 \& t2 & 10000 & A3 \& $\mathrm{t} 1$ & \begin{tabular}{|l|}
11000 \\
\end{tabular} & A4 \& t2 \\
\hline 000001 & A1 \& t3 & 01001 & A2 \& t4 & 10001 & A3 \& t3 & $\frac{11000}{11001}$ & A4 \& t4 \\
\hline 00010 & A2 \& t5 & 01010 & A1 \& t6 & 10010 & A4 \& t5 & 11010 & \begin{tabular}{|l|} 
A3 \& $\& 6$ \\
\end{tabular} \\
\hline 00011 & A2 \& 17 & 01011 & A1 \& t8 & 10011 & A4 \& t7 & 11011 & A3 \& t8 \\
\hline 0 & A1 \& 17 & & A2 \& t8 & 10100 & A3 \& +7 & $\frac{1101}{11100}$ & \begin{tabular}{|l|l}
$A 4 \&$ \& 8 \\
\end{tabular} \\
\hline 00101 & A1\& \& 5 & 01101 & A2 \& t6 & 10101 & A3 \& t5 & $\frac{11100}{11101}$ & \begin{tabular}{|l|} 
A4 \& \& t6 \\
\end{tabular} \\
\hline & $A 2 \& t 3$ & 01110 & A1 \& t4 & 10110 & A4 \& $t 3$ & $\frac{1111}{11110}$ & A3 \& t4 \\
\hline 00111 & A2 \& t1 & 01111 & A1 \& t2 & 10111 & A4 \& t1 & 11111 & A3 \& t2 \\
\hline \multicolumn{8}{|c|}{ Gray Pulse Mapping Table } \\
\hline \begin{tabular}{|l} 
Input Data Bits \\
( 5 bits per \\
symbol)
\end{tabular} & \begin{tabular}{|c|} 
Assigned \\
Amplitude \& \\
Time position
\end{tabular} & $\begin{array}{l}\text { Input Data Bits } \\
\text { ( } 5 \text { bits per } \\
\text { symbol) }\end{array}$ & \begin{tabular}{|c|} 
Assigned \\
Amplitude \& \\
Time position
\end{tabular} & $\begin{array}{l}\text { Input Data Bits } \\
\text { ( } 5 \text { bits per } \\
\text { symbol) }\end{array}$ & \begin{tabular}{|c|} 
Assigned \\
Amplitude \& \\
Time position
\end{tabular} & \begin{tabular}{|l} 
Input Data Bits \\
( 5 bits per \\
symbol $)$
\end{tabular} & \begin{tabular}{|c|} 
Assigned \\
Amplitude \& \\
Time position
\end{tabular} \\
\hline 00000 & A1 \& t1 & 01000 & A1 \& $\mathrm{t} 8$ & 10000 & A4 \& $\mathrm{t} 1$ & 11000 & A4 \& 18 \\
\hline & A2 \& t1 & 01 & A2 \& t8 & 100 & A3 \& t1 & 11001 & A3 \& t8 \\
\hline 00010 & A1 \& $\mathrm{t} 2$ & 01010 & A1 \& $\mathrm{t} 7$ & 10010 & A4 \& $\mathrm{t} 2$ & 11010 & A4 \& $t 7$ \\
\hline 00011 & A2 \& t2 & 01011 & A2 \& 77 & 10011 & A3 \& t2 & 11011 & A3 \& t7 \\
\hline 00100 & A1 \& t4 & 01100 & A1 \& t5 & 10100 & A4 \& t4 & 11100 & A4 \& t5 \\
\hline 00100 & A1 \& \& t4 & 01100 & A1 \& 15 & $\frac{10100}{10101}$ & A3 \& t4 & $\frac{11100}{11101}$ & \begin{tabular}{|l|}
$A 4 \& 15$ \\
\& \& t5
\end{tabular} \\
\hline 00110 & A1 \& t3 & 01110 & A1 \& t6 & 10110 & A4 \& 33 & 11110 & A4 \& t6 \\
\hline 00111 & A2 \& $\mathrm{t} 3$ & 01111 & A2 \& t6 & 10111 & A3 \& $\mathrm{t} 3$ & 11111 & A3 \& t6 \\
\hline
\end{tabular}

In Table 1, all neighboring pulses have a fixed Hamming distance " 1 " at Gray mapping table. However, the Hamming distance between any two neighboring pulses of proposed mapping table is either " 3 " or " 4 ". For example, the pulse with amplitude $A_{3}$ and $t_{7}$ has a Hamming distance " 3 ” from two neighboring pulses and " 4 " from another two neighboring pulses. Therefore, the overall minimum Hamming distance is " 3 ", which is larger than " 1 ” of Gray mapping.

The proposed mapping method is not restricted only to above combination of $M$-ary PPM and $N$-ary PAM. The method can be employed to a combination of any two of pulse amplitude, pulse position, pulse polarity and pulse phase. If a TR scheme is involved, the amplitude, time position, polarity and phase of data pulse related to the reference pulse also can be used in the combination.

\section{Simulation Results}

In this chapter, the simulation results are presented to show the performance of proposed Hamming distance enlarging pulse mapping method over AWGN channel. In the simulation, a convolutional encoder with code rate $3 / 5$ and constraint length 3 is adopted, and the Trellis decoding algorithm is employed at the receiver. The transmission power (symbol energy) and the source input rate are fixed. When convolutional coding with coding rate $3 / 5$ is used, every 3 bits are coded to be 5 bits at the same symbol duration. Thus the modulation order has to be increased and a combination of 8-PPM and 4-PAM is used, where each pulse represents $\log _{2}(8 \times 4)=5$ bits.

The proposed and Gray mapping tables in Table 1 are compared by computer simulation. As shown in Figure 6, when convolutional coding is involved, the proposed mapping method brings a smaller BER value than the Gray mapping. For example, at the BER of $10^{-2}$, the SNR of $12 \mathrm{~dB}$ is required for proposed mapping, which is much smaller than $16 \mathrm{~dB}$ for Gray mapping. Thus, the system architect can adopt the proposed pulse mapping method with FEC codes to achieve the best system performance.

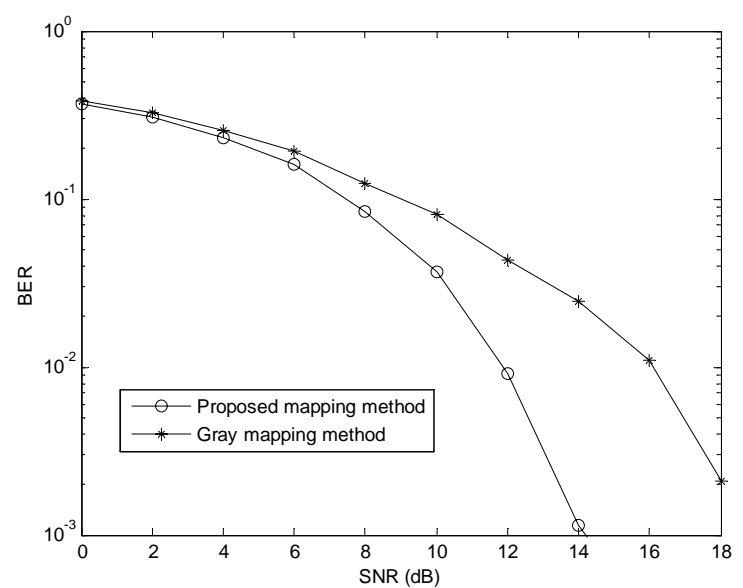

Figure 6 BER vs SNR for various mapping methods with convolutional coding

\section{Conclusions}

In this paper, a novel Hamming distance enlarging pulse mapping method is proposed for the high-order IR modulation. The proposed pulse mapping method can maximize the Hamming distance between all neighboring pulses of IR UWB systems. Thus the proposed pulse mapping method can effectively achieve a smaller BER than the conventional Gray mapping, when the channel coding is involved. This has been proven by our simulation results in this paper.

\section{References}

[1] M.Z. Win and R.A. Scholtz, "Impulse radio: How it works,” IEEE Comm. Letters, vol.2, pp. 36-38, Feb. 1998.

[2] M.Z. Win and R.A. Scholtz, "Ultra-wide bandwidth timehopping spread spectrum impulse radio for wireless multiple-access communications," IEEE Trans. Commun. vol. 48, pp. 679-691, Apr. 2000.

[3] IEEE TG3a official website: http://www.ieee802.org/15/pub/TG3a.html

[4] IEEE TG3c official website: http://www.ieee802.org/15/pub/TG3c.html

[5] N. Rinaldi, et. al., "U.C.A.N.'s ultra wide band system: baseband algorithm design,” Proc. of IWUWBS, Finland, 25 June 2003.

[6] J. G. Proakis, Digital Communications, $3^{\text {rd }}$ ed. New York: McGraw-Hill, 1995.

[7] M. Di Benedetto and G. Giancola, Understanding Ultra Wide Band Radio Fundamentals, NJ: Prentice Hall, 2004.

[8] R. Hoctor and H. Tomlinson, "An overview of delayhopped, transmitted-reference RF communications,” GE Technical Report 2001 CRD198, Jan. 2002.

[9] R. Hoctor, et. al., "Ultra-wideband communications system”, US patent application 2001/0053175, General Electric company.

[10] T. Zasowski, et. al., "An energy efficient transmittedreference scheme for ultra-wideband communications," Proc. of IWUWBS and UWBST, pp. 146-150, Japan, 18-21 May 2004. 